www.nature.com/ja

\title{
OBITUARY
}

\section{Professor Emeritus C Richard Hutchinson (10 October 1943-5 January 2010)}

The Journal of Antibiotics (2010) 63, 409-410; doi:10.1038/ja.2010.67

$\mathrm{C}$ Richard 'Dick'/'Hutch' Hutchinson, PhD, Edward Leete Professor Emeritus of Medicinal Chemistry, and Professor Emeritus of Bacteriology, died on Tuesday, 5 January 2010 at the age of 66 after a courageous fight against cancer.

Professor Hutchinson was born on 10 October 1943 in Dayton, OH, USA. He attended Ohio State University (BS, 1966) and the University of Minnesota (PhD, 1970). After postdoctoral work at Cambridge University, UK (1971), he was an Assistant Professor of Pharmacognosy (1971-1974), University of Connecticut, and Assistant Professor (1974-1977), Associate Professor (1977-1982), Professor (1982-2000), Edward Leete Professor (1995-2000) of Medicinal Chemistry and Professor of Bacteriology (1986-2000), all at University of Wisconsin-Madison. He retired from University of Wisconsin-Madison in 2000 to join Kosan Biosciences, Hayward, CA, USA as a Vice President New Technologies. He returned part-time to the University of Wisconsin-Madison in 2004, left Kosan in December 2006 and co-founded Centrose LLC, Madison, WI, USA, in 2007. He was most recently President and Chief Scientific Officer of Centrose LLC and continued his ad hoc teaching and research advising at the University.

During a distinguished career that spanned nearly four decades at the University of Wisconsin-Madison, Kosan Biosciences and Centrose LLC, Professor Hutchinson trained many future academicians and industrial leaders, touched many lives with his wisdom, made major advances in understanding the biosynthesis of naturally occurring drugs through studies of the molecular genetics and biochemistry of antibiotic production in microorganisms, and worked tirelessly and enthusiastically through creative entrepreneurship to translate laboratory findings into clinical products.

Professor Hutchinson's early training and independent work were in chemistry, wherein he made many important contributions to alkaloid biosynthesis. Even to this day his studies on camptothecin represent most of what we know about the biosynthesis of this important clinical compound. To later generations of natural products practitioners Professor Hutchinson is better known as one of the world leaders who elegantly blended the art and science of chemistry, biochemistry and molecular biology to understand secondary metabolite biosynthesis in actinomycetes, in particular the biosynthesis of polyketide and deoxysugar natural products.

Professor Hutchinson has made seminal contributions to both type I and type II polyketide synthases (PKSs). For type II PKSs, he is best known for his work on the tetracenomycin $(\mathrm{Tcm})$ and daunorubicin (Dnr) biosynthesis gene clusters. Legacies of his work include: (i) the tcm and $d n r$ clusters as two of the best characterized type II PKS gene

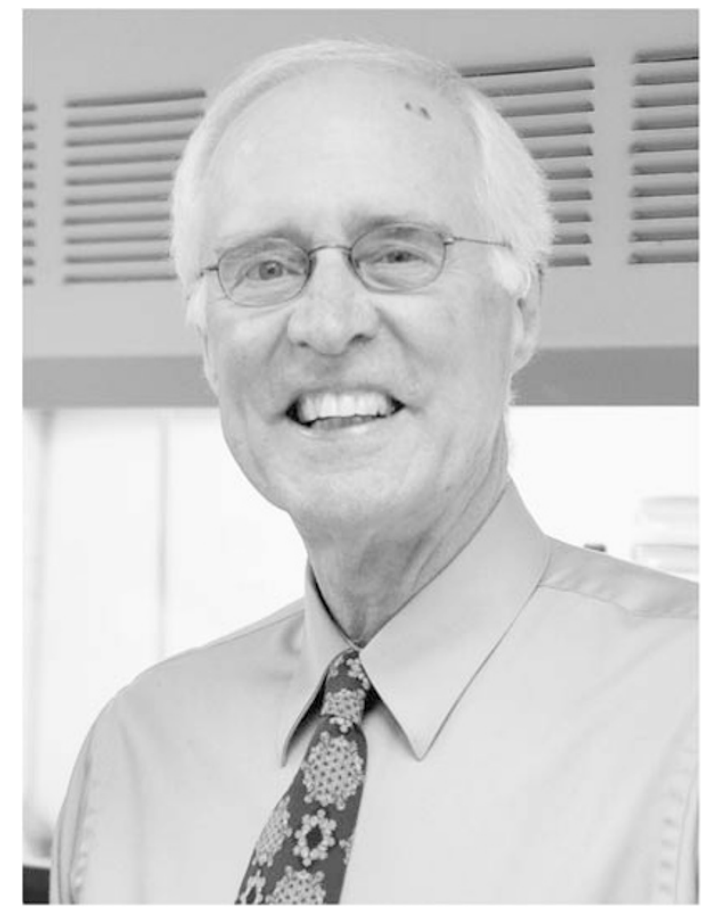

clusters to date, serving as models for aromatic polyketide biosynthesis in general; (ii) in vitro reconstitution and characterization of the Tcm PKS opened the possibility to study the enzyme mechanism and structure of type II PKS in vitro; and (iii) cross-talk between fatty acid biosynthesis and Tcm biosynthesis in Streptomyces glaucescens led to the identification and subsequent confirmation of the malonyl CoA:acyl carrier protein transferase as the missing link between fatty acid (primary metabolism) and polyketide biosynthesis (secondary metabolism). For type I PKSs, he is best known for his work on tylosin and rifamycin (Rif) biosynthesis gene clusters. Highlights from his work in this field include: (i) his hypothesis and demonstration by feeding experiments that type I PKS acts by a processive mechanism, which was widely considered as a milestone for macrolide biosynthesis; this conceptual advance was critical for the subsequent discovery and characterization of the modular type I PKS and (ii) characterization of the rif cluster and isolation of a family of elongating polyketide intermediates experimentally confirmed the first time the processive mechanism of type I PKS. 
Professor Hutchinson was a pioneer of combinatorial biosynthesis for natural product structural diversity. The most notable contribution of his laboratory was the development of methodologies, strategies and concepts dictating effective combinatorial biosynthesis. Highlights from his laboratory include: (i) demonstration that the ketoacyl synthase $\alpha$ - and $\beta$ - subunits of type II PKS both contribute to the chain length for aromatic polyketide biosynthesis; (ii) recognition that synthesis of aromatic polyketides bearing starter units other than acetate requires dedicated enzymes that interact with the type II PKS complex; (iii) exploration of environmental DNAs for the production of complex natural products; and (iv) the production of epirubicin, a commercially important clinical drug, by a metabolically engineered organism, the only example known to date that has been practically advanced by combinatorial biosynthesis.

Professor Hutchinson also made seminal contributions to the study of fungal polyketide biosynthesis. Recognizing that fungal PKSs are mechanistically and structurally distinct from bacterial PKSs and that fungal polyketides represent a major source of clinically important natural products, Professor Hutchinson in the middle 1990s started to work on lovastatin biosynthesis in Aspergillus terreus as a model system for polyketide biosynthesis in fungi. The cloning, sequencing and characterization of lovastatin gene cluster unveiled a novel type of PKS and set the stage for the engineering and production of novel lovastatin analogs, a class of antihypercholesterolemic drugs currently with a market exceeding $\$ 10$ billion annually.

Reflective of his work on important clinical drugs such as daunorubicin and lovastatin, Professor Hutchinson helped to create the mold for what is now termed 'translational research'. In addition, he was integral to the operations of numerous pharmaceutical companies, initially in advisory roles (he was the chair of the Scientific Advisor Board for TerraGen) and subsequently in leadership roles such as Vice President of Kosan Biosciences and President and Chief Scientific Officer of Centrose LLC.

Professor Hutchinson's enthusiasm for natural products extended far beyond his group's contributions, as evidenced by his sharing of various tools developed by his group with uncounted members of the scientific community. To give just one example, pWHM-series
(WHM stands for Wisconsin, Hutchinson and Madison) vectors are commonly used by scientists all over the globe and greatly facilitate the cloning and engineering of secondary metabolite biosynthesis in Streptomyces.

Professor Hutchinson published over 240 papers, held numerous federal and industrial research grants, served on the editorial boards of many peer-reviewed journals, including the Journal of Antibiotics (1983-2006) and received many prestigious awards, including the Guggenheim and Fulbright Fellowships, the Charles Thom Research Achievement Award of the Society for Industrial Microbiology, the AACP Paul Dawson Biotechnology Award, the Research Achievement Award of the American Society for Pharmacognosy and a Distinguished Alumni Award from Ohio State University.

Professor Hutchinson was an extraordinary teacher and mentor who trained and mentored a generation of young scientists in chemistry, biochemistry and molecular biology of secondary metabolite biosynthesis. Many of these people are now successful scientists at both academic and industrial institutions nationally and internationally. Through the effect of their discoveries on natural products biosynthesis and drug discovery, Professor Hutchinson's legacy of scientific rigor, progressivism and standard of excellence will be continued.

Professor Hutchinson will be remembered for his strength of spirit, zeal for life and new experiences, and his boundless intellectual curiosity. He has left an indelible mark, not only on our field of science, but even more so on the lives of those lucky enough to have learned from him and who considered him a friend and a role model. $\mathrm{He}$ is sorely missed by all who knew him but for having known him we are better scientists and better people. For that, we are forever grateful.

Ben Shen

Division of Pharmaceutical Sciences, School of Pharmacy, and Department of Chemistry, University of Wisconsin-Madison,

Madison, WI, USA

E-mail: bshen@pharmacy.wisc.edu 